\title{
Temperature dependence of the local structure and lattice dynamics of wurtzite-type $\mathrm{ZnO}$
}

\author{
J. Timoshenko a, A. Anspoks a , A. Kalinko ${ }^{\mathrm{b}}$, A. Kuzmin ${ }^{\mathrm{a}}$ \\ ${ }^{a}$ Institute of Solid State Physics, University of Latvia, Kengaraga street 8, LV-1063 Riga, \\ Latvia \\ ${ }^{b}$ Synchrotron SOLEIL, l'Orme des Merisiers, Saint-Aubin, BP 48, 91192 Gif-sur-Yvette,
} France

\begin{abstract}
Temperature-dependent (10-300 K) Zn K-edge EXAFS spectra for polycrystalline wurtzite-type $\mathrm{ZnO}$ were analysed using ab initio multiple-scattering theory and taking into account anisotropy of the crystallographic structure and thermal disorder. We employed two different simulation approaches: classical molecular dynamics (MD) and reverse Monte Carlo coupled with evolutionary algorithm (RMC/EA method). The accuracy of several forcefield models, which are commonly used in the MD simulations of bulk and nanostructured $\mathrm{ZnO}$, was tested based on a comparison between the experimental and simulated Zn K-edge EXAFS spectra. It was found that available force-field models fail to describe accurately many-atom distribution functions. More accurate solution was obtained by the RMC/EA method, which allowed us also to resolve the non-equivalent groups of atoms in the first two coordination shells around absorbing $\mathrm{Zn}$ atom and to follow the changes of structural parameters with temperature variation. It was found that upon increasing temperature the structure of $\mathrm{ZnO}$ becomes more anisotropic due to the increase of internal parameter $u$ of the oxygen Wyckoff position (2b) and related $\mathrm{Zn}_{0}-\mathrm{O}_{2}$ distances.
\end{abstract}

Keywords: ZnO; EXAFS; reverse Monte Carlo simulations; classical molecular dynamics simulations

Email addresses: janis.timoshenko@gmail.com (J. Timoshenko), a.kuzmin@cfi.lu.lv (A. Kuzmin)

URL: http://www.cfi.lu.1v/ () 


\section{Introduction}

Zinc oxide $(\mathrm{ZnO})$ is a wide band-gap $\left(E_{g}=3.37 \mathrm{eV}[1]\right)$ semiconductor, which has many promising technological applications $[2,3,4,5]$ and, therefore, is an extremely popular research field during the last years $[6,7,8]$. Crystalline lattice of $\mathrm{ZnO}$ in the most common wurtzite-type phase is essentially anisotropic $[9,10,11]$ that is reflected in its piezoelectric $[12,13]$ and pyroelectric [14] properties. The wurtzite structure has a hexagonal unit cell with two lattice parameters, $a$ and $c$, and belongs to the space group of $P 6_{3} m c$ (No. 186) [2]. It is composed of two interpenetrating hexagonal-closepacked sublattices, each built up by one type of atoms ( $\mathrm{Zn}$ or O), which are displaced with respect to each other along the threefold $c$-axis by the amount of internal parameter $u$. The $\mathrm{Zn}$ and $\mathrm{O}$ atoms are in the $(2 \mathrm{~b})$ Wyckoff positions: Zn $(0,0,0),(1 / 3,2 / 3,1 / 2)$; O $(0,0, u),(1 / 3,2 / 3,1 / 2+u)$. Both $\mathrm{Zn}$ and $\mathrm{O}$ atoms are tetrahedrally coordinated by four atoms of the other type, and the value of the internal parameter $u$ controls the degree of the $\mathrm{ZnO}_{4}$ tetrahedra distortion, $u c$ corresponds to the distance between average positions of nearest $\mathrm{Zn}$ and $\mathrm{O}$ atoms in the [0001] direction.

The anisotropy of the $\mathrm{ZnO}$ lattice directly influences its lattice dynamics, which is also anisotropic, as revealed by X-ray diffraction measurements of thermal expansion and thermal ellipsoids along $a$ and $c$ directions $[15,16,17]$. Obviously, for the design of different nanostructured ZnO-based materials the deep comprehension of such anisotropic properties is essential. However, even in the case of bulk $\mathrm{ZnO}$ material the understanding of the local atomic structure and lattice dynamics is still far from complete [18, 19].

This issue can be addressed by the extended X-ray absorption fine-structure (EXAFS) spectroscopy, which is a powerful, element-specific technique [20] suitable for the investigations of local atomic structure in a broad class of materials (single crystals and polycrystalline powders, nanostructured and amorphous materials, even liquids and molecules [21]). Rapid evolution of synchrotron radiation sources during the last decades led to significantly improved quality of EXAFS spectra that potentially allows one to extract larger amount of structural information. While the nearest coordination shells around absorber can be accessed by conventional EXAFS analysis [22] within the single-scattering approximation, the treatment of contributions from the outer shells and many-atom distribution functions requires advanced approaches, based on the multiple-scattering (MS) theory [20]. This is a challenging task, whose solution could give the access to the information on 
distributions of bonding angles and, noteworthy, anisotropy of the material structure $[23,24,25]$. In the case of $\mathrm{ZnO}$ this means that thermal disorder and interatomic distances for at least the first three coordination shells, containing nearest oxygen and zinc atoms within the $a b$-plane and along the $c$-axis direction, should be determined with high enough accuracy.

To achieve this goal, it is essential to properly account for the MS contributions into the total Zn K-edge EXAFS spectrum: as it will be shown below, these contributions are significant for the outer shells. Such analysis is challenging due to a large amount of model parameters, if the conventional approach, based on the multi-shell fit [20], is used.

Until now only a few EXAFS studies of ZnO have been carried out. The analysis of the first coordination shell around absorbing $\mathrm{Zn}$ atom was performed in $[26,27,28]$. It was extended recently up to the third shell of $\mathrm{Zn}$ in pure $\mathrm{ZnO}$ thin films grown at different nitrogen partial pressures in [29]. Pressure-dependent Zn K-edge EXAFS spectra were analysed in [30], taking into account some multiple-scattering contributions. A number of studies have been devoted to the investigations of doped $\mathrm{ZnO}$ thin films and different nanosystems (nanorods, nanoparticles, nanowires). For example, the local environment around $\mathrm{Fe}[31]$, $\mathrm{Co}[32,33], \mathrm{Ni}[34,35]$ and $\mathrm{Cu}[28,36,37]$ in $\mathrm{ZnO}$ has been investigated, based on the analysis of the dopant metal $\mathrm{K}$-edge X-ray absorption spectra. An excellent review on the applications of $\mathrm{x}$-ray absorption spectroscopy for the investigation of nanostructured $\mathrm{ZnO}$ can be found in [1]. Finally, the study of the O K-edge in single-crystal ZnO has been also carried out in [38].

In this study we propose to use simulation-based techniques for the analysis of temperature-dependent (10-300 K) Zn K-edge EXAFS data from polycrystalline wurtzite-type $\mathrm{ZnO}$, taking into account all important MS contributions, anisotropy of the system and also the thermal disorder effects. Two techniques, classical molecular dynamics (MD) [39] and reverse Monte Carlo method coupled with evolutionary algorithm (RMC/EA) [40, 41], have been employed for the interpretation of EXAFS spectra with the goals (i) to advance the understanding of the local atomic structure and dynamics of $\mathrm{ZnO}$ lattice and (ii) to validate existing force-field models [42, 43, 44] commonly used in MD simulations. Note that preliminary room temperature results have been presented by us recently in [45]. 


\section{Experimental and data analysis}

X-ray absorption spectra of wurtzite-type ZnO (99.99\%, Alfa Aesar) were acquired at the Zn K-edge in transmission mode at the HASYLAB/DESY $\mathrm{C}$ bending-magnet beamline [46]. The X-ray beam intensity before and after the sample was measured by two ionization chambers filled with a mixture of argon and krypton gases. The energy scan of the incident radiation was sustained by the double-crystal monochromator $\mathrm{Si}(111)$, detuned to remove higher-order harmonics. Polycrystalline $\mathrm{ZnO}$ powder sample was deposited on Millipore filter and fixed by Scotch tape. The sample thickness was optimized to obtain the absorption $\mathrm{Zn}$ K-edge jump value $\Delta \mu \approx 1$. A liquid He flow cryostat was used to perform temperature dependent measurements in the range between 10 and $300 \mathrm{~K}$.

The experimental Zn K-edge EXAFS spectra were extracted using the conventional procedure $[22,47]$ and are shown together with their Fourier transforms (FT) in Fig. 1. The first peak in FT at $1.5 \AA$ is due to the contribution of the first coordination shell (four closest oxygen atoms) around the absorbing zinc atom, whereas the second main peak at $2.9 \AA$ originates mainly due to the outer coordination shells formed by zinc atoms. Due to the overlap of contributions from different coordination shells and due to the mentioned contributions of many-atom distribution functions (the so-called multiple-scattering effects), the analysis of EXAFS data beyond the first coordination shell is a complicated task. Moreover, as it will be shown below, in the case of $\mathrm{ZnO}$ even the first coordination shell consists of two separate contributions, which cannot be separated using conventional EXAFS analysis due to the small difference $(\sim 0.03 \AA)$ of two non-equivalent $\mathrm{Zn}-\mathrm{O}$ bond lengths within the $\mathrm{ZnO}_{4}$ tetrahedra $[9,10,11]$. To treat these problems, we used two different numerical approaches, based on classical MD and RMC simulations.

\section{MD-EXAFS simulations}

In classical MD one needs to specify empirical force-field model to calculate interatomic forces. Then the classical Newton's equations of motion can be integrated over time, and the trajectories of atoms can be accumulated. As a result, thousands of different atomic configurations are obtained. For each configuration the EXAFS is calculated using ab initio multiplescattering theory [20], and the configuration-averaged EXAFS spectrum is obtained [39] and directly compared with the experimental spectrum. 
The MD simulations were performed by the GULP code [48, 49] in the canonical (NVT) ensemble with periodic boundary conditions. The simulation box had a size of the $5 a \times 5 a \times 3 c$ relaxed supercell $(a=3.2706 \AA$, $c=5.1379 \AA$ ), containing 300 atoms. The Newton's equations of motion were integrated with the Verlet leapfrog algorithm [50], with a time step of 0.5 fs. The Nosé-Hoover thermostat [51] was used to keep the average temperature around $T=300 \mathrm{~K}$ during simulations.

Three common force-field models for $\mathrm{ZnO}$, all based on the Buckinghamtype potential, were taken from $[42,43,44]$. Formal ion charges $Z(\mathrm{Zn})=+2$ and $Z(\mathrm{O})=-2$ were used in the calculations of Coulomb interaction. Note that the values of parameters in the Buckingham potentials were chosen to have the best possible agreement between calculated and experimentally measured lattice constants, elastic and dielectric properties in $[42,44]$ and also between corresponding phonon spectra in [43]. For each force-field model a set of 4000 static atomic configurations was generated during a simulation run of 20 ps after system equilibration for 20 ps. These configurations were further used to calculate the Zn K-edge configuration-averaged EXAFS signal (MD-EXAFS) using ab initio real-space multiple-scattering FEFF8 code [52]. The multiple-scattering contributions were accounted up to the 7th order. The calculation of the cluster potential was performed only once for the average atom configuration, corresponding to the $\mathrm{ZnO}$ crystallographic structure. The complex exchange-correlation Hedin-Lundqvist potential and default values of muffin-tin radii $\left(R_{\mathrm{mt}}(\mathrm{Zn})=1.27 \AA\right.$ and $\left.R_{\mathrm{mt}}(\mathrm{O})=1.0 \AA\right)$, as provided within the FEFF8 code [52], were employed.

Before discussing the results of the MD-EXAFS simulations, we need to emphasize that in conventional $\mathrm{MD}$ for $\mathrm{ZnO}$ we cannot incorporate the socalled shell model, as used in the original works $[43,44]$. Below we will illustrate, how such simplification influences the lattice dynamics of $\mathrm{ZnO}$. The results of our lattice dynamics calculations within the harmonic approximation are compared with the experimental data [19], obtained by inelastic neutron scattering and Raman spectroscopy, in Fig. 2(a). As one can see, when the shell is excluded from the force-field model, some optical branches become shifted to higher frequencies, as one can expect. At the same time, acoustic and low lying optical branches, located below $600 \mathrm{~cm}^{-1}$, are in a reasonable agreement with the experimental values. At this point, the question arises whether such difference in the high frequency modes will affect the amplitudes of relative thermal vibrations, which are relevant for the description of our EXAFS spectra. As it was shown previously in [53, 54], the high- 
frequency modes actually have very little effect on the radial distribution function and, thus, on the vibration amplitudes.

Within the harmonic approximation, the mean square relative displacement (MSRD) of the two atoms depends on the eigenvalues and eigenvectors of dynamical matrix through the integral over all normal modes [55]. However, the contribution of each normal mode is inversely proportional to the square of its frequency. Therefore, optical modes are relatively less important than acoustic ones. We have calculated the MSRD values as a function of temperature for $\mathrm{Zn}-\mathrm{O}$ and $\mathrm{Zn}-\mathrm{Zn}$ atom pairs in $\mathrm{ZnO}$ using the Kulkarni's force-field model [42] following the procedure described in [56]. The obtained results are reported in Fig. 2(b). As one can see, neglecting the modes above $600 \mathrm{~cm}^{-1}$ gives no effect in the case of $\mathrm{Zn}-\mathrm{Zn}$ atom pair and results in a small decrease $\left(\sim 0.0003 \AA^{2}\right)$ of the MSRD values for $\mathrm{Zn}-\mathrm{O}$ atom pairs. Thus, we concluded that excluding the shell from the force-field models during the MD run should not influence significantly the final results since the highfrequency vibration modes produce little effect on the amplitude of relative thermal vibrations and, thus, also on the EXAFS amplitude.

The results of the MD-EXAFS simulations using three different force-field models $[42,43,44]$ are compared with the experimental Zn K-edge EXAFS spectrum in Fig. 3. As one can see, all three force-field models are able to reproduce rather well the behavior of the zinc coordination shells up to about $4.5 \AA$, but they fail for outer shells. In particular, the peaks in FT at $\sim 4.6 \AA$ and $\sim 6.1 \AA$ (indicated by arrows in Fig. 3 ) have much higher amplitude in the simulation than in the experiment. However, the detailed analysis of the first two peaks in FT at $\sim 1.5 \AA$ and $\sim 2.9 \AA$ (Fig. 3) suggests that also for the nearest coordination shells the accuracy of the force-field models is not sufficient to describe properly atom dynamics due to the model simplicity. Note that on average, the model from [42] is slightly better than the other two $[43,44]$.

The significant differences between the experimental Zn K-edge EXAFS and MD-EXAFS spectra beyond $4.5 \AA$ in FT can be attributed to the influence of MS effects, related to the many-atom distribution functions [23]. In Fig. 4 the total contributions of the single-scattering (SS), double-scattering (DS) and triple-scattering (TS) events are shown separately. As one can see, the MS (DS and TS) signals dominate in the $R$-space ranges where the largest disagreement between the experimental EXAFS and MD-EXAFS spectra is observed, i.e., at 4.5-4.8 $\AA$ and 5.6-6.6 $\AA$. Besides, there is also a prominent DS contribution at $3.1 \AA$ (under the second peak in FT) and combined DS 
and TS contribution at $5.3 \AA$. Note that the MS contributions are substantially out-of-phase with the SS signals, thus leading to the reduction of the total EXAFS amplitude.

To conclude, the force-field models from $[42,43,44]$ are able to describe the average structure of $\mathrm{ZnO}$ but fail to reproduce accurately its lattice dynamics, i.e., the amplitudes of atom vibrations and correlation effects.

\section{RMC/EA-EXAFS calculations}

Reverse Monte Carlo simulation of EXAFS is potentially capable to achieve better agreement with the experimental data than the MD-EXAFS method due to the possibility to fine tune the structural model. Also, opposite to classical molecular dynamics, the RMC method is applicable at low temperatures. We have followed the procedure, described in [40], but the conventional RMC scheme [57] has been modified using the evolutionary algorithm (EA) [41]. Now instead of just one atomic configuration several supercells are modeled simultaneously. Exchange of information between these supercells via selection and crossover operators allows one to explore the configuration space more efficiently, thus making the whole simulation much less demanding for computing resources. In this study 32 supercells with a size of $4 a \times 4 a \times 4 c(a=3.2496 \AA, c=5.2042 \AA$ [11] $)$, containing 256 atoms each, have been used in simulations. Comparison of the experimental and theoretical EXAFS spectra has been performed using Morlet wavelet transform (WT) $[58,59]$ in the $k$-space range from $3 \AA^{-1}$ to $15 \AA^{-1}$ and in the $R$-space range from $0.9 \AA$ to $5.5 \AA$. Being a straightforward mathematical procedure as the Fourier transform [60], the WT allows one to obtain a two-dimensional representation of the signal with simultaneous localization in $k$ and $R$ spaces that is an advantage over conventional fit in $k$ or $R$ space only.

In Fig. 5 the supercell, used in the RMC calculations, and the obtained total and partial RDFs for Zn-O and Zn-Zn atom pairs are shown. The calculated Zn K-edge EXAFS spectra and their Fourier transforms, corresponding to the final RMC configurations (RMC/EA-EXAFS), are compared with the experimental data at four representative temperatures in Fig. 6. Note that the RMC/EA-EXAFS spectrum includes contributions from the scattering paths with the half-path length up to $6.9 \AA$ and the multiple-scattering contributions up to the third order. As one can see, good agreement between the experimental and calculated Zn K-edge EXAFS spectra is achieved both in $k$ and $R$ space, also in the ranges (4.5-4.8 $\AA$ and 5.6-6.6 $\AA$ ), where the 
MD-EXAFS models fail (Fig. 3). This result suggests that the structural model, obtained by the RMC/EA method, is reliable enough to be used in further analysis for the extraction of more detailed information.

\section{Discussion}

\subsection{Comparison of $M D$ and $R M C / E A$ results}

First, we will discuss the origin of the problems in the case of the MD models. In Fig. 7 (left panel) we show separately the MS and SS contributions to the Fourier transform of the total RMC/EA-EXAFS spectrum. As one can see, here the contribution of the MS effects at 4.5-4.8 $\AA$ is much smaller than in the case of the MD models. Besides, the main interlayer (along $c$ axis direction) $\mathrm{SS}\left(\mathrm{Zn}_{0} \rightleftarrows \mathrm{Zn}, R=6.14 \AA\right.$ ) and intralayer (along $a$ or $b$ axis direction) $\mathrm{TS}\left(\mathrm{Zn}_{0} \rightleftarrows \mathrm{Zn} \leftrightarrows \mathrm{Zn}, R=6.50 \AA\right.$ ) signals contributing at 5.6-6.6 in the FT are comparable in the RMC/EA model, whereas the TS signal dominates strongly the SS one in the MD models due to the difference in the thermal damping factors (see Fig. 7 (right panels)). Therefore, we conclude that the main problem of the MD models $[42,43,44]$ is related to the proper accounting for atom vibrations.

The partial radial distribution functions (RDFs) for $\mathrm{Zn}-\mathrm{O}$ and $\mathrm{Zn}-\mathrm{Zn}$ atom pairs, obtained from the MD simulations using the force-field model by Kulkarni [42] and from the RMC/EA analysis, are compared in Fig. 5 (left panel). At first glance, they are quite close, but differ in details. To highlight the differences, we show (middle and right panels in Fig. 5) separately contributions into the partial RDFs from non-equivalent oxygen $\left(\mathrm{O}_{1}\right.$ and $\left.\mathrm{O}_{2}\right)$ and zinc $\left(\mathrm{Zn}_{1}\right.$ and $\left.\mathrm{Zn}_{2}\right)$ atoms, located in the first and second coordination shells of absorbing $\mathrm{Zn}_{0}$ atom, respectively. As one can see, the $\mathrm{Zn}-\mathrm{Zn}$ RDFs obtained by the two models are quite close. However, there is clear difference in the amplitude between the $\mathrm{Zn}_{0}-\mathrm{O}_{1}$ contributions from oxygen atoms located in the $a b$-plane. Also the width and position of the $\mathrm{Zn}_{0}-\mathrm{O}_{2}$ peaks, due to oxygen atoms located along the $c$-axis, deviate appreciably. The failure of the MD simulations comes from the simplicity of the $\mathrm{Zn}-\mathrm{O}$ pair potential model [42]: it is not able to reproduce accurately the non-centrosymmetric $\mathrm{ZnO}$ crystal structure $[9,10,11]$, which is responsible for its piezoelectricity.

The partial RDFs, obtained by the RMC/EA method, were used to evaluate the MSRD values for the $\mathrm{Zn}-\mathrm{O}$ and $\mathrm{Zn}-\mathrm{Zn}$ atom pairs. At room temperature they are equal to $\sigma^{2}\left(\mathrm{Zn}_{0}-\mathrm{O}_{1}\right)=0.0040 \pm 0.0003 \AA^{2}$ and $\sigma^{2}\left(\mathrm{Zn}_{0}-\mathrm{Zn}_{1}\right)=$ $0.0067 \pm 0.0006 \AA^{2}$ along the $c$-axis and to $\sigma^{2}\left(\mathrm{Zn}_{0}-\mathrm{O}_{2}\right)=0.0042 \pm 0.0009 \AA^{2}$ 
and $\sigma^{2}\left(\mathrm{Zn}_{0}-\mathrm{Zn}_{2}\right)=0.0088 \pm 0.0009 \AA^{2}$ in the orthogonal direction (in the $a b$-plane). These MSRD values indicate that the interactions within $\mathrm{Zn}-\mathrm{O}$ and $\mathrm{Zn}-\mathrm{Zn}$ atom pairs differ along the $c$-axis and in the $a b$-plane. We will address this difference in details in the next section.

\subsection{Influence of temperature}

The RMC/EA-EXAFS method was successfully employed to perform accurate analysis of the Zn K-edge EXAFS spectra for crystalline $\mathrm{ZnO}$ at the temperatures $T \leq 300 \mathrm{~K}$ (Fig. 6). Good reproduction of all peaks in FTs up to $7 \AA$ gives us a confidence in the structural information extracted from the outer coordination shells. The final RMC configurations were used to calculate temperature dependencies of the average $\mathrm{Zn}-\mathrm{O}$ and $\mathrm{Zn}-\mathrm{Zn}$ interatomic distances and corresponding MSRD factors, characterizing the amplitudes of relative atomic oscillations (Fig. 8).

According to the x-ray diffraction data $[10,11]$ (see dashed lines in Fig. 8), the average distances $\langle R\rangle_{\mathrm{D}}\left(\mathrm{Zn}_{0}-\mathrm{Zn}_{1}\right)$ and $\langle R\rangle_{\mathrm{D}}\left(\mathrm{Zn}_{0}-\mathrm{Zn}_{2}\right)$ should vary little solely due to the lattice thermal expansion. However, they grow with increasing temperature, as observed by EXAFS, due to the additional contribution caused by thermal vibrations [61]. Note that the average interatomic ZnZn distance probed by EXAFS $\langle R\rangle_{\mathrm{EXAFS}}=\langle R\rangle_{\mathrm{D}}+\left\langle\left(\Delta \sigma_{\perp}\right)^{2}\right\rangle / 2\langle R\rangle_{\mathrm{D}}$ should be systematically larger than that $\langle R\rangle_{\mathrm{D}}$ probed in diffraction experiments $[10,11]$ due to the second term accounting for the perpendicular MSRD $\left\langle\left(\Delta \sigma_{\perp}\right)^{2}\right\rangle$ of the neighboring atom in the direction orthogonal to the interatomic bond [61, 62].

At the same time, the average distances between zinc and four nearest oxygen atoms, forming $\mathrm{ZnO}_{4}$ tetrahedron (Fig. 5), vary differently with increasing temperature. The average distance $\langle R\rangle_{\operatorname{EXAFS}}\left(\mathrm{Zn}_{0}-\mathrm{O}_{2}\right)$ between the absorbing zinc and the apical oxygen atom, located along the $c$-axis, increases, whereas the average distance $\langle R\rangle_{\text {EXAFS }}\left(\mathrm{Zn}_{0}-\mathrm{O}_{1}\right)$ between the absorbing zinc and the three basal oxygen atoms, located within the $a b$-plane, is slightly reduced. This result implies that the internal parameter $u$, giving the equilibrium position of oxygen atoms along the $c$-axis [63], varies with temperature. The values of the parameter $u$ as a function of temperature, calculated from the final RMC configurations, are shown in the bottom left panel in Fig. 8. It can be seen that the parameter $u$ increases by about 0.01 with increasing temperature from 10 to $300 \mathrm{~K}$. Clearly, this conclusion should be supported by the diffraction experiments. Surprisingly, to our knowledge up to now there exists only one low temperature study of the parameter $u$ 
by neutron diffraction at $20 \mathrm{~K}$ [17]. It was concluded in [17] that below room temperature the parameter $u$ does not change significantly. The contradiction between our results, obtained from EXAFS analysis, and neutron diffraction data in [17] is especially intriguing, since it is known that the parameter $u$ is responsible for the pyroelectric properties of wurtzite-type compounds [64].

Temperature dependencies of the parallel MSRD's $\sigma^{2}$ for the four atom pairs $\left(\mathrm{Zn}_{0}-\mathrm{O}_{1}, \mathrm{Zn}_{0}-\mathrm{O}_{2}, \mathrm{Zn}_{0}-\mathrm{Zn}_{1}\right.$ and $\left.\mathrm{Zn}_{0}-\mathrm{Zn}_{2}\right)$ were fitted using correlated Einstein model [65] (bottom right panel in Fig. 8). The obtained values of the Einstein frequency $(\omega)$ were used to estimate effective bond-strength constants $(k)$. They are equal to $\omega\left(\mathrm{Zn}_{0}-\mathrm{O}_{1}\right)=83 \pm 9 \mathrm{THz}, \omega\left(\mathrm{Zn}_{0}-\mathrm{O}_{2}\right)=71 \pm$ $15 \mathrm{THz}, \omega\left(\mathrm{Zn}_{0}-\mathrm{Zn}_{1}\right)=27 \pm 1 \mathrm{THz}, \omega\left(\mathrm{Zn}_{0}-\mathrm{Zn}_{2}\right)=22 \pm 1 \mathrm{THz}$ and $k\left(\mathrm{Zn}_{0}-\right.$ $\left.\mathrm{O}_{1}\right)=147 \pm 33 \mathrm{~N} \mathrm{~m}^{-1}, k\left(\mathrm{Zn}_{0}-\mathrm{O}_{2}\right)=108 \pm 28 \mathrm{~N} \mathrm{~m}^{-1}, k\left(\mathrm{Zn}_{0}-\mathrm{Zn}_{1}\right)=79 \pm 8 \mathrm{~N}$ $\mathrm{m}^{-1}, k\left(\mathrm{Zn}_{0}-\mathrm{Zn}_{2}\right)=54 \pm 5 \mathrm{~N} \mathrm{~m}^{-1}$. The obtained results suggest that the atoms, located within one $a b$-plane (i.e., $\mathrm{Zn}_{0}-\mathrm{Zn}_{1}$ and $\mathrm{Zn}_{0}-\mathrm{O}_{1}$ atom pairs) are interacting stronger than the atoms, located in the neighboring layers along the $c$-axis $\left(\mathrm{Zn}_{0}-\mathrm{Zn}_{2}\right.$ and $\mathrm{Zn}_{0}-\mathrm{O}_{2}$ atom pairs), although the distances between, correspondingly, $\mathrm{Zn}_{0}-\mathrm{Zn}_{1}$ and $\mathrm{Zn}_{0}-\mathrm{Zn}_{2}$, and $\mathrm{Zn}_{0}-\mathrm{O}_{1}$ and $\mathrm{Zn}_{0}-\mathrm{O}_{2}$ are very close. Thus it is clear that such system in principle cannot be described accurately by simple pairwise force-field models. Note also that the differences between atom pairs $\mathrm{Zn}_{0}-\mathrm{Zn}_{1}$ and $\mathrm{Zn}_{0}-\mathrm{Zn}_{2}$, and $\mathrm{Zn}_{0}-\mathrm{O}_{1}$ and $\mathrm{Zn}_{0}-\mathrm{O}_{2}$, have been already observed in the case of oriented wurtzite-type $\mathrm{ZnO}$ nanorods in [66], where the polarization of the synchrotron X-ray beam has been exploited to discriminate contributions of different atom pairs. However, to our knowledge, such strikingly different behavior is established for the first time for the bulk $\mathrm{ZnO}$ in this study.

Finally, we would like to comment on the MSRD value for the $\mathrm{Zn}_{0}-\mathrm{O}_{2}$ bond at $10 \mathrm{~K}$, which is significantly smaller than the one expected from the Einstein model fit to higher temperature experimental data (Fig. 8). This fact can be related to very low vibration amplitude of Zn atoms observed previously by neutron diffraction at $20 \mathrm{~K}$ in [17]. The origin of such behavior is unclear and requires further investigations.

\section{Conclusions}

The local atomic structure and lattice dynamics in polycrystalline wurtzitetype $\mathrm{ZnO}$ were studied by the $\mathrm{Zn} \mathrm{K}$-edge X-ray absorption spectroscopy in the temperature range from 10 to $300 \mathrm{~K}$. Temperature dependent EXAFS spectra 
were analysed using two advanced theoretical approaches based on classical molecular dynamics (MD-EXAFS) and reverse Monte Carlo/evolutionary algorithm (RMC/EA-EXAFS) methods.

The MD-EXAFS method allowed us to use the experimental EXAFS spectra to validate the existing force-field models [42, 43, 44]. We found that the three models are able to reproduce general behavior of the lattice dynamics in bulk $\mathrm{ZnO}$ but fail in the accurate description of thermal motion, especially, the many-atom correlations. Therefore, one should take care when applying them to nanostructured zinc oxide.

The RMC/EA-EXAFS method gives more accurate description of the thermal disorder in $\mathrm{ZnO}$, since it is not limited by any simplified model. As a result, it allowed us to discriminate contributions of non-equivalent $\mathrm{Zn}$ and $\mathrm{O}$ atoms in the first and second coordination shells around the absorbing $\mathrm{Zn}$ atoms. Essential differences between $\mathrm{Zn}-\mathrm{O}$ and $\mathrm{Zn}-\mathrm{Zn}$ bonds aligned along the $c$-axis and in the $a b$-plane of $\mathrm{ZnO}$ hexagonal crystal lattice were revealed and are responsible for its noncentrosymmetric structure. Our results suggest that both $\mathrm{Zn}$ and $\mathrm{O}$ atoms, located within one $a b$-plane, interact stronger than the atoms at approximately the same distance, but located in the neighboring layers along the $c$-axis. The observed changes of the average distances $\mathrm{Zn}_{0}-\mathrm{O}_{1}$ and $\mathrm{Zn}_{0}-\mathrm{O}_{2}$ (Fig. 8) also imply that with increasing temperature the structure of $\mathrm{ZnO}$ becomes more anisotropic, since the oxygen $\mathrm{O}_{2}$ is pulled closer to the upper $a b$-plane (corresponding internal parameter $u$ of the oxygen Wyckoff position (2b) and related $\mathrm{Zn}_{0}-\mathrm{O}_{2}$ distances increase). The latter result needs to be confirmed by diffraction measurements.

\section{Acknowledgments}

The work was supported by European Social Fund within the projects 2009/0138/1DP/1.1.2.1.2/09/IPIA/VIAA/004 ("Support for Doctoral Studies at University of Latvia") and 2013/0015/1DP/1.1.1.2.0/13/APIA/VIAA/010. The EXAFS experiments at HASYLAB/DESY were supported by the EC FP7 under grant agreement No. 226716.

\section{References}

[1] Mino L, Agostini G, Borfecchia E, Gianolio D, Piovano A, Gallo E, et al. J Phys D: Appl Phys 2013;46:423001. 
[2] Özgür U, Alivov YI, Liu C, Teke A, Reshchikov MA, Doğan S, et al. J Appl Phys 2005;98:041301.

[3] Willander M, Nur O, Zhao QX, Yang LL, Lorenz M, Cao BQ, et al. Nanotech 2009;20:332001.

[4] Hochbaum AI, Yang P. Chem Rev 2010;110:527.

[5] King PDC, Veal TD. J Phys: Condens Matter 2011;23:334214.

[6] Pearton S, Norton D, Ip K, Heo Y, Steiner T. Prog Mat Sci 2005;50:293.

[7] Singh S, Thiyagarajan P, Mohan Kant K, Anita D, Thirupathiah S, Rama N, et al. J Phys D: Appl Phys 2007;40:6312.

[8] Janotti A, Van de Walle CG. Rep Prog Phys 2009;72:126501.

[9] Abrahams SC, Bernstein JL. Acta Crystall B 1969;25:1233.

[10] Reeber RR. J Appl Phys 1970;41:5063.

[11] Karzel H, Potzel W, Köfferlein M, Schiessl W, Steiner M, Hiller U, et al. Phys Rev B 1996;53:11425.

[12] Dal Corso A, Posternak M, Resta R, Baldereschi A. Phys Rev B 1994;50:10715.

[13] Wang ZL, Song J. Science 2006;312:242.

[14] Yang Y, Guo W, Pradel KC, Zhu G, Zhou Y, Zhang Y, et al. Nano Lett 2012;12:2833.

[15] Schulz H, Thiemann K. Solid State Commun 1979;32:783.

[16] Kihara K, Donnay G. Can Mineral 1985;23:647.

[17] Albertsson J, Abrahams S, Kvick Å. Acta Crystallogr B 1989;45:34.

[18] Shalimov A, Paszkowicz W, Grasza K, Skupiski P, Mycielski A, BakMisiuk J. Phys Stat Sol B 2007;244:1573.

[19] Serrano J, Manjón FJ, Romero AH, Ivanov A, Cardona M, Lauck R, et al. Phys Rev B 2010;81:174304. 
[20] Rehr JJ, Albers RC. Rev Mod Phys 2000;72:621.

[21] Yano J, Yachandra V. Photosynth Res 2009;102:241.

[22] Aksenov V, Kovalchuk M, Kuzmin A, Purans Y, Tyutyunnikov S. Cryst Rep 2006;51:908.

[23] Filipponi A, Di Cicco A. Phys Rev B 1995;52:15135.

[24] Di Cicco A, Trapananti A, Faggioni S, Filipponi A. Phys Rev Lett 2003;91:135505.

[25] Di Cicco A, Trapananti A. J Phys: Condens Matter 2005;17:S135.

[26] Yoshiasa A, Maeda H, Ishii T, Emura S, Moriga T, Koto K. Solid State Ionics 1995;78:31.

[27] Nedoseikina T, Shuvaev A, Vlasenko V. J Phys: Condens Matter 2000;12:2877.

[28] Ma Q, Prater JT, Sudakar C, Rosenberg RA, Narayan J. J Phys: Condens Matter 2012;24:306002.

[29] Haug J, Chassé A, Dubiel M, Eisenschmidt C, Khalid M, Esquinazi P. J Appl Phys 2011;110:063507.

[30] Decremps F, Datchi F, Saitta A, Polian A, Pascarelli S, Di Cicco A, et al. Phys Rev B 2003;68:104101.

[31] Wei XX, Song C, Geng KW, Zeng F, He B, Pan F. J Phys: Condens Matter 2006;18:7471.

[32] Lu ZL, Hsu HS, Tzeng Y, Zhang FM, Du YW, Huang JCA. Appl Phys Lett 2009;95:102501.

[33] Bin-Bin L, Hong-Lie S, Rong Z, Xiang-Qiang X, Zhi X. Chin Phys Lett 2007;24:3473.

[34] Venkataiah G, Huang MR, Su H, Liu C, Huang J. J Phys Chem C 2010;114:16191.

[35] Mino L, Gianolio D, Bardelli F, Prestipino C, Kumar ES, Bellarmine F, et al. J Phys: Condens Matter 2013;25:385402. 
[36] Ma Q, Buchholz DB, Chang RPH. Phys Rev B 2008;78:214429.

[37] Vachhani PS, Dalba G, Ramamoorthy RK, Rocca F, Šipr O, Bhatnagar AK. J Phys: Condens Matter 2012;24:506001.

[38] Tröger L, Yokoyama T, Arvanitis D, Lederer T, Tischer M, Baberschke K. Phys Rev B 1994;49:888.

[39] Kuzmin A, Evarestov R. J Phys: Condens Matter 2009;21:055401.

[40] Timoshenko J, Kuzmin A, Purans J. Comp Phys Commun 2012;183:1237.

[41] Timoshenko J, Kuzmin A, Purans J. J Phys: Condens Matter 2014;26:055401.

[42] Kulkarni A, Zhou M, Ke F. Nanotech 2005;16:2749.

[43] Zaoui A, Sekkal W. Phys Rev B 2002;66:174106.

[44] Lewis G, Catlow C. J Phys C: Solid State Phys 1985;18:1149.

[45] Timoshenko J, Anspoks A, Kalinko A, Kuzmin A. Phys Status Solidi C 2014;:DOI: 10.1002/pssc.201300615.

[46] Rickers K, Drube W, Schulte-Schrepping H, Welter E, Brüggmann U, Herrmann M, et al. AIP Conf Proc 2007;882:905.

[47] Kuzmin A. Physica B 1995;208-209:175.

[48] Gale JD. Phil Mag B 1996;73:3.

[49] Gale JD, Rohl AL. Mol Simul 2003;29:291.

[50] Hockney RW. Methods Comput Phys 1970;9:136.

[51] Hoover WG. Phys Rev A 1985;31:1695.

[52] Ankudinov A, Ravel B, Rehr J, Conradson S. Phys Rev B 1998;58:7565.

[53] Goodwin AL, Tucker MG, Cope ER, Dove MT, Keen DA. Phys Rev B 2005;72:214304. 
[54] Goodwin AL, Tucker MG, Cope ER, Dove MT, Keen DA. Physica B 2006;385:285.

[55] Chung JS, Thorpe MF. Phys Rev B 1997;55:1545.

[56] Cope ER, Dove MT. J Appl Crystallogr 2007;40:589.

[57] McGreevy R, Pusztai L. Mol Simul 1988;1:359.

[58] Muñoz M, Argoul P, Farges F. Am Mineral 2003;88:694.

[59] Timoshenko J, Kuzmin A. Comp Phys Commun 2009;180:920.

[60] Tao Q, Mang VI, Yuesheng X, editors. Wavelet Analysis and Applications. Basel: Birkhäuser Verlag; 2007.

[61] Fornasini P. J Phys: Condens Matter 2001;13:7859.

[62] Busing WR, Levy HA. Acta Crystallogr 1964;17:142.

[63] Kisi EH, Elcombe MM. Acta Crystallogr C 1989;45:1867.

[64] Mair SL, Barnea Z. Acta Crystallogr A 1975;31:201.

[65] Sevillano E, Meuth H, Rehr JJ. Phys Rev B 1979;20:4908.

[66] Han SW, Yoo HJ, An SJ, Yoo J, Yi GC. Appl Phys Lett 2005;86:021917. 

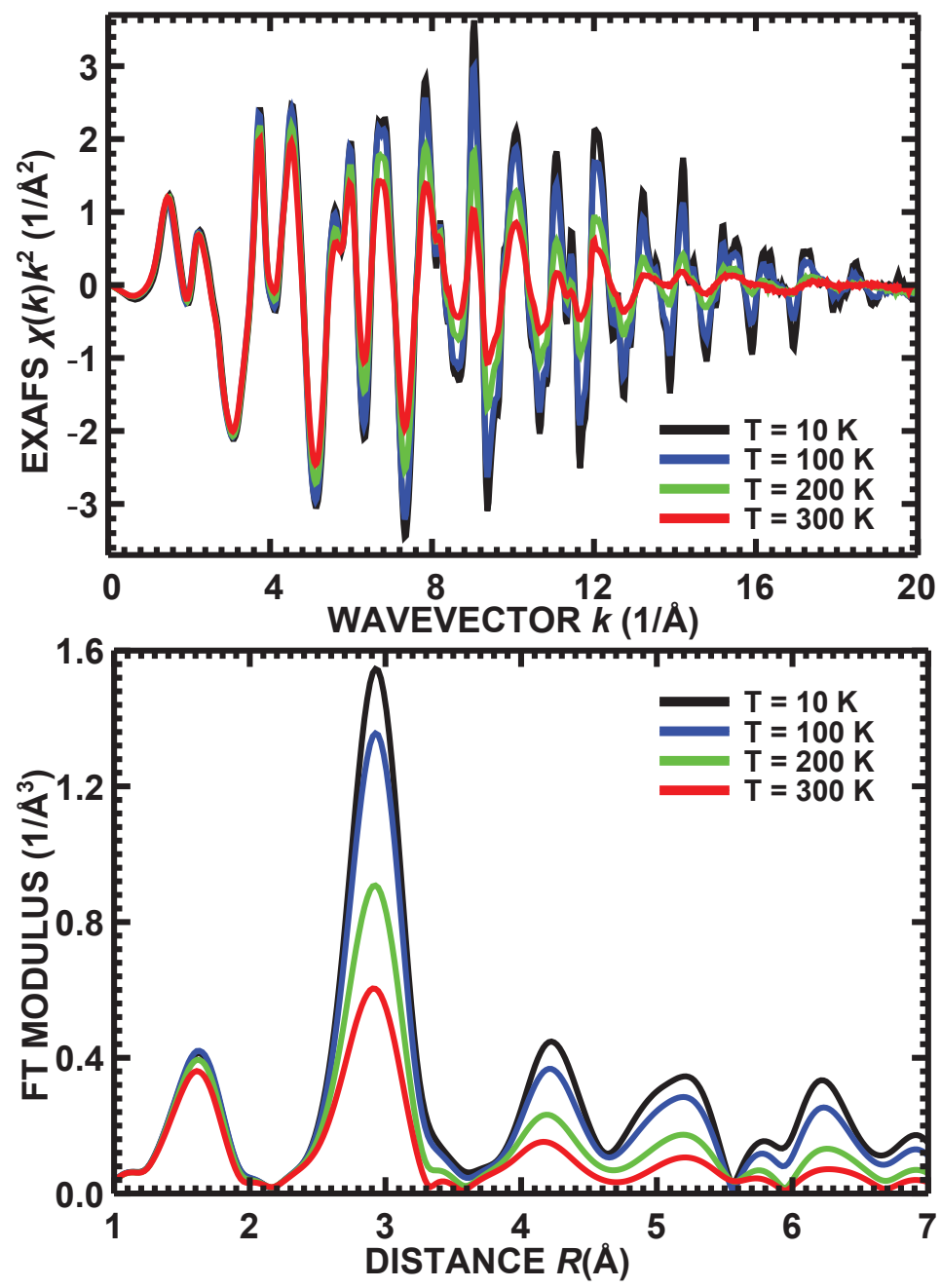

Figure 1: (Color online) Experimental Zn K-edge EXAFS spectra $\chi(k) k^{2}$ and their Fourier transforms (FT) for polycrystalline $\mathrm{ZnO}$ measured at temperatures from 10 to $300 \mathrm{~K}$. 

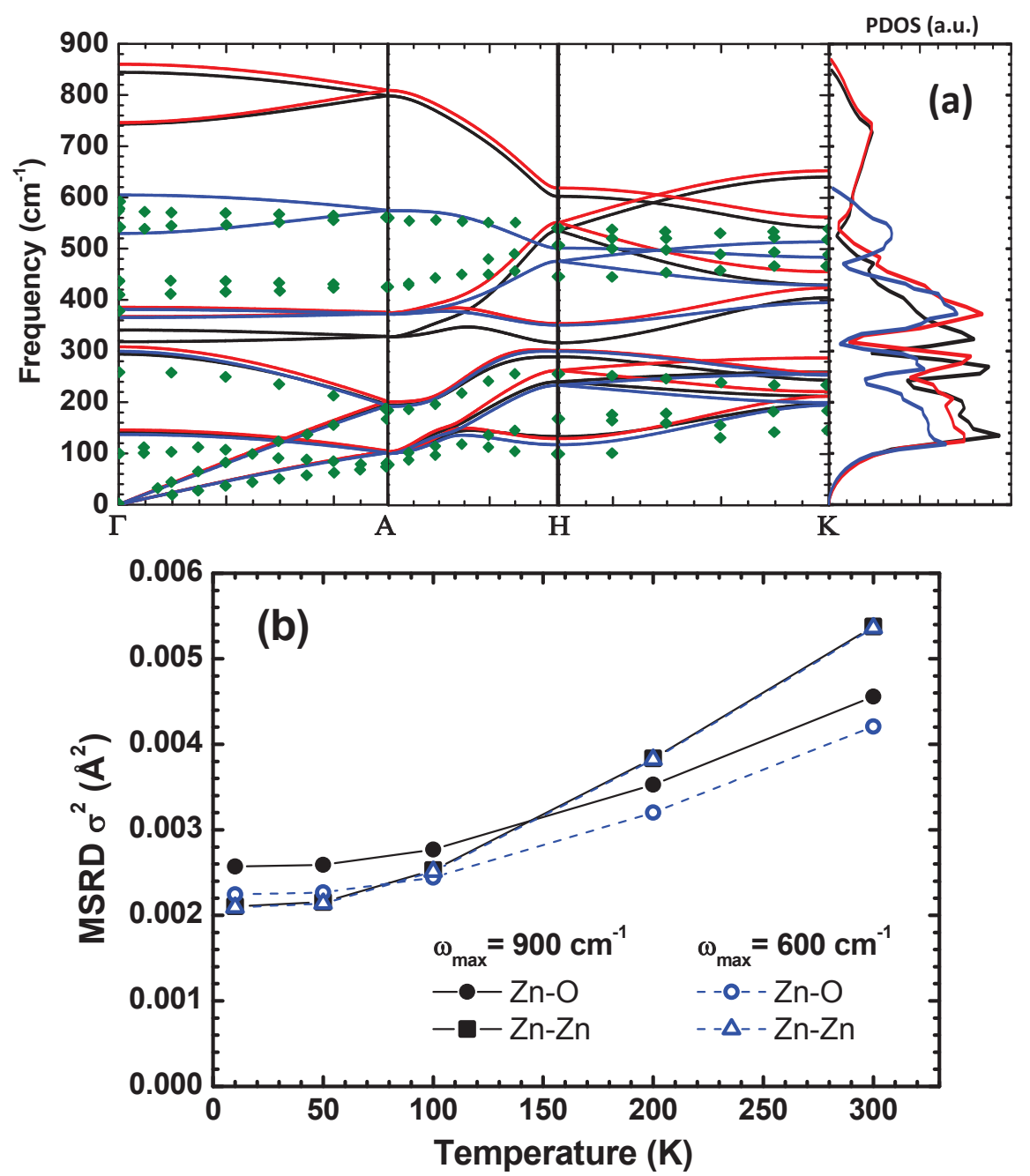

Figure 2: (Color online) (a) Phonon-dispersion relations of $\mathrm{ZnO}$ along the main symmetry directions and phonon density of states (PDOS). Solid blue lines corresponds to the shell model from Zaoui [43]; solid black and red lines correspond to the models without shell from Kulkarni [42] and Zaoui [43], respectively. Experimental inelastic neutron scattering and Raman scattering data from [19] are shown by diamonds. (b) Temperature dependence of the MSRD $\sigma^{2}$ for $\mathrm{Zn}-\mathrm{O}$ and $\mathrm{Zn}-\mathrm{Zn}$ atom pairs calculated taking into account all modes up to $\omega_{\max }=900 \mathrm{~cm}^{-1}$ (solid circles and squares) and neglecting the modes above $\omega_{\max }=600 \mathrm{~cm}^{-1}$ (open circles and triangles). Note that the results for $\mathrm{Zn}-\mathrm{Zn}$ atom pair coincide. 


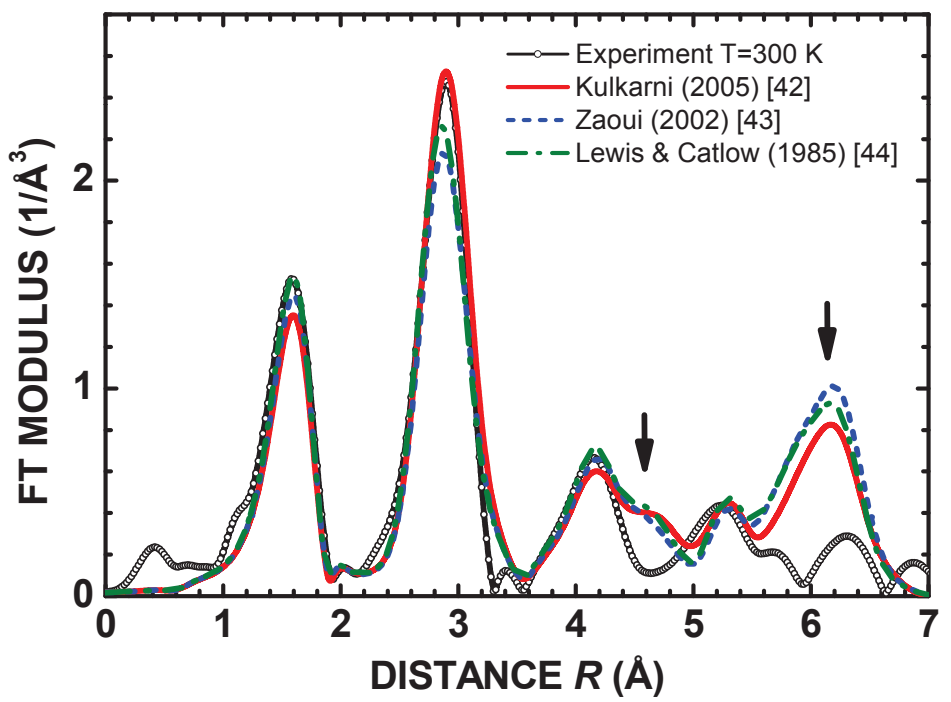

Figure 3: (Color online) Fourier transforms of the experimental and MD-EXAFS Zn Kedge EXAFS spectra $\chi(k) k^{2}$ for polycrystalline ZnO. The MD-EXAFS spectra were calculated using three different force-field models [42, 43, 44]. 

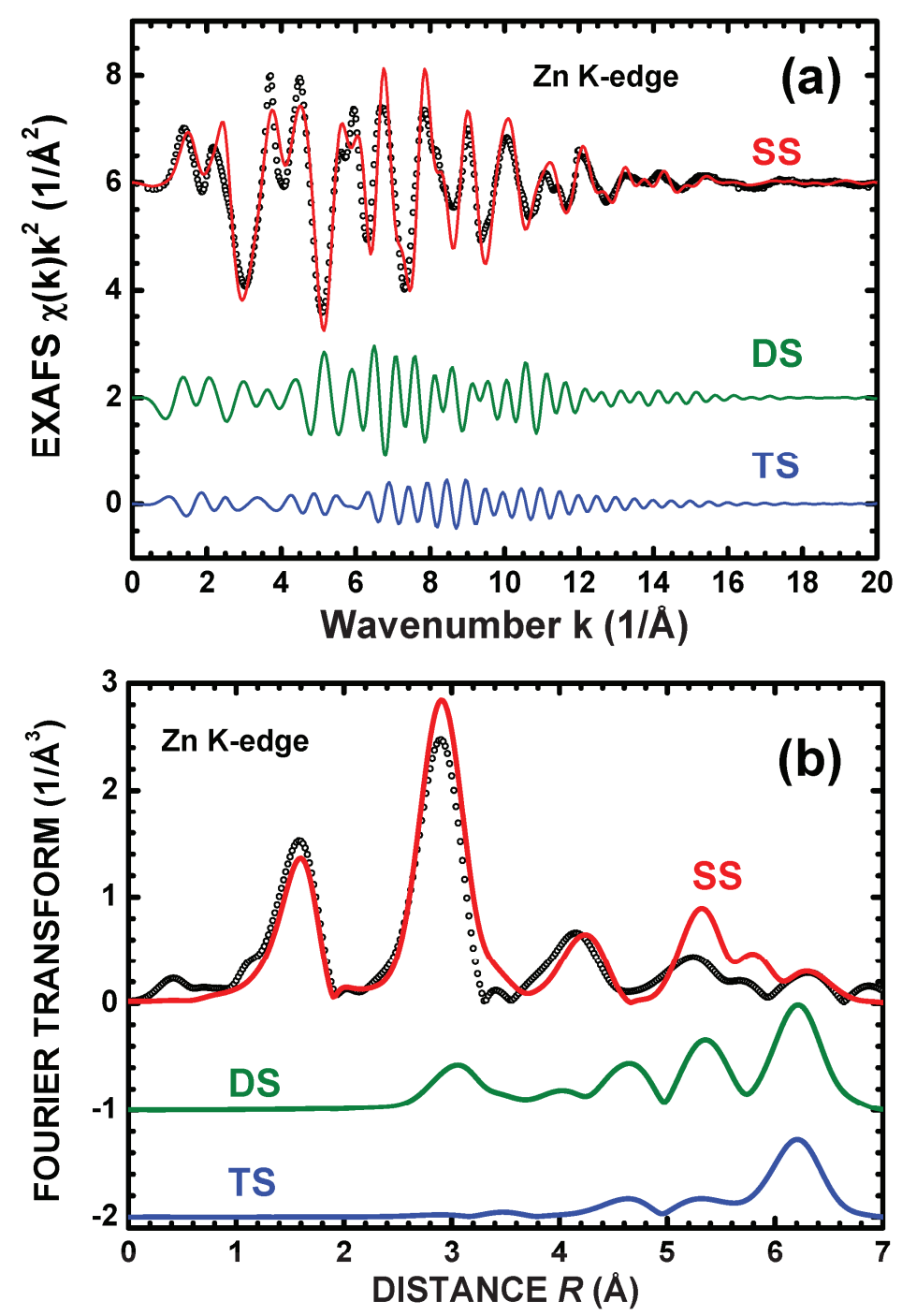

Figure 4: (Color online) Single-scattering (SS), double-scattering (DS) and triplescattering (TS) contributions into the Zn K-edge MD-EXAFS spectrum $\chi(k) k^{2}$, obtained with the force-field model from [42], and their Fourier transforms. The experimental EXAFS spectrum for polycrystalline $\mathrm{ZnO}$, measured at $T=300 \mathrm{~K}$, is shown by open circles. The spectra are shifted vertically for clarity. 

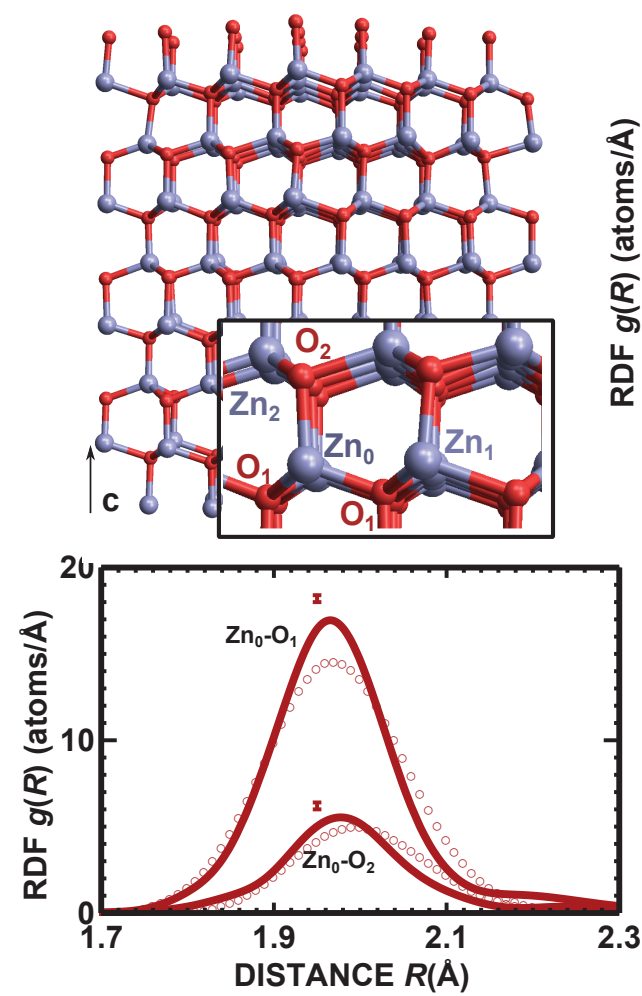
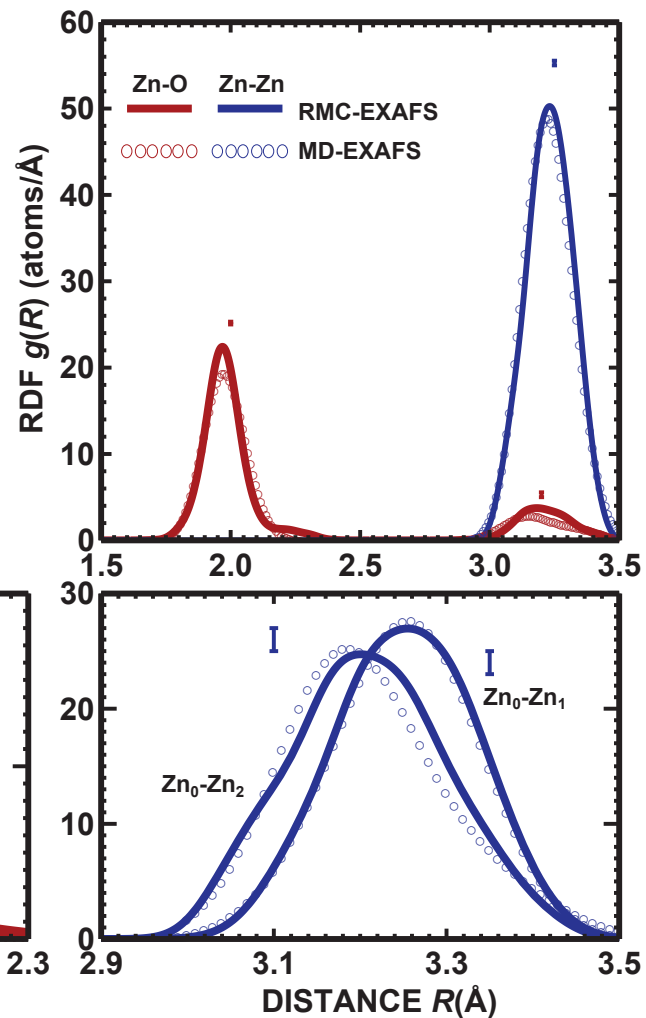

Figure 5: (Color online) Upper left panel: supercell used in the RMC/EA simulation (blue large balls are zinc atoms, red small balls are oxygen atoms). Upper right panel: partial radial distribution functions (RDFs) for $\mathrm{Zn}-\mathrm{O}$ (red lines) and $\mathrm{Zn}-\mathrm{Zn}$ (blue lines) atom pairs, obtained from the MD simulations with the force-field model by Kulkarni [42] (circles) and from the RMC/EA analysis (solid lines) at $T=300 \mathrm{~K}$. Lower panels: contributions into RDFs from non-equivalent oxygen and zinc atoms, located in the first and second shells, respectively. 

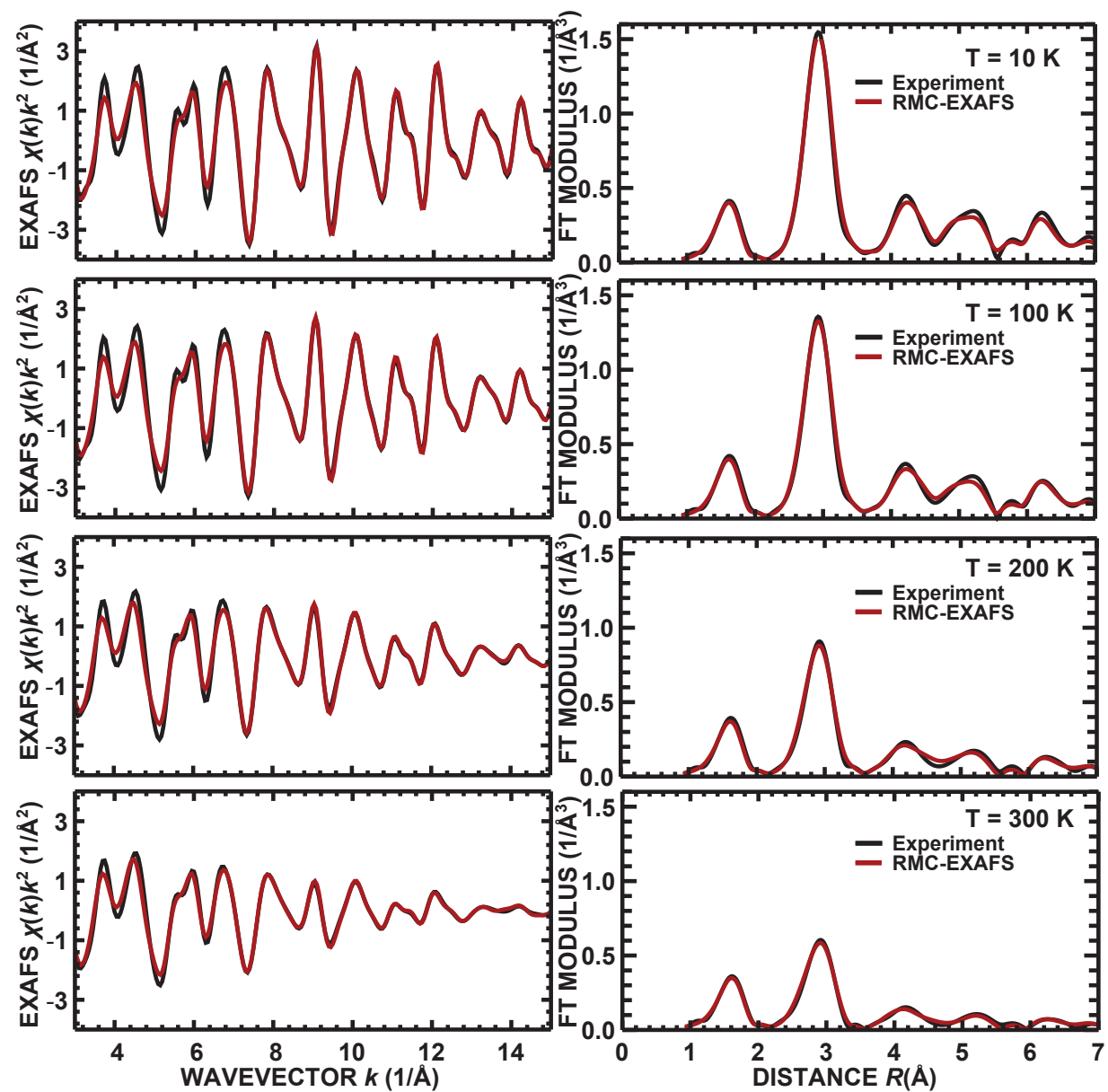

Figure 6: (Color online) Results of the RMC/EA-EXAFS calculations for the experimental $\mathrm{Zn} \mathrm{K}$-edge EXAFS spectra of polycrystalline $\mathrm{ZnO}$ at four representative temperatures. 


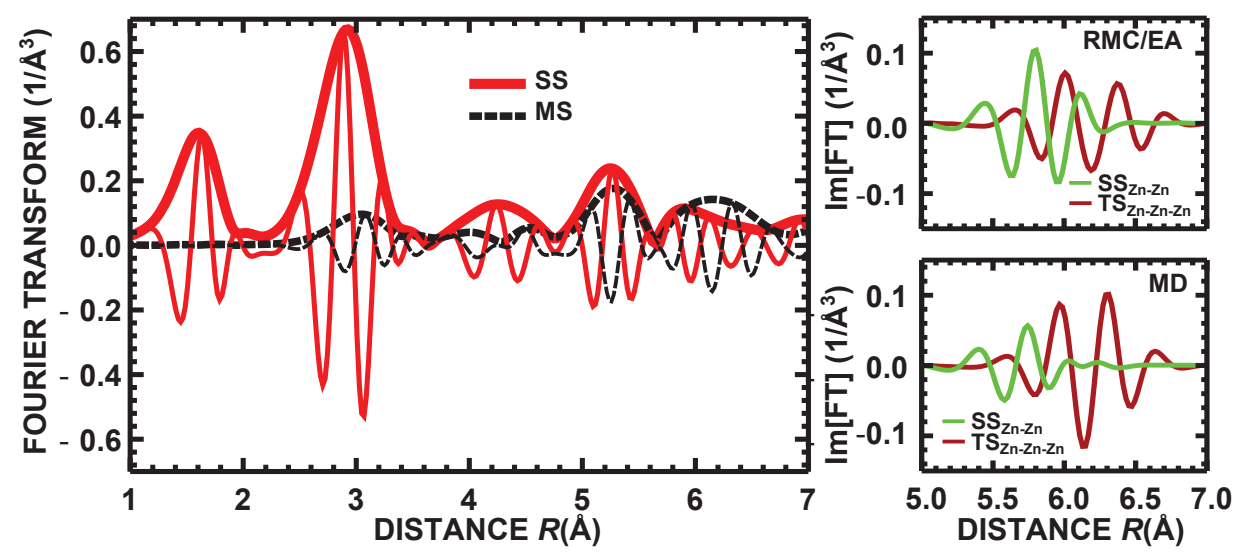

Figure 7: (Color online) Left panel: Fourier transforms (imaginary parts (thin lines) and moduli (thick lines)) of the total SS and MS contributions to the Zn K-edge RMC/EAEXAFS spectra, obtained from the best fit of the experimental data for polycrystalline $\mathrm{ZnO}$ measured at $T=300 \mathrm{~K}$. Right panels: Imaginary parts of the Fourier transforms of the $\mathrm{SS}\left(\mathrm{Zn}_{0} \rightleftarrows \mathrm{Zn}, R=6.14 \AA\right)$ and $\mathrm{TS}\left(\mathrm{Zn}_{0} \rightleftarrows \mathrm{Zn} \leftrightarrows \mathrm{Zn}, R=6.50 \AA\right)$ EXAFS spectra calculated by $\mathrm{RMC} / \mathrm{EA}$ and $\mathrm{MD}$ methods. 

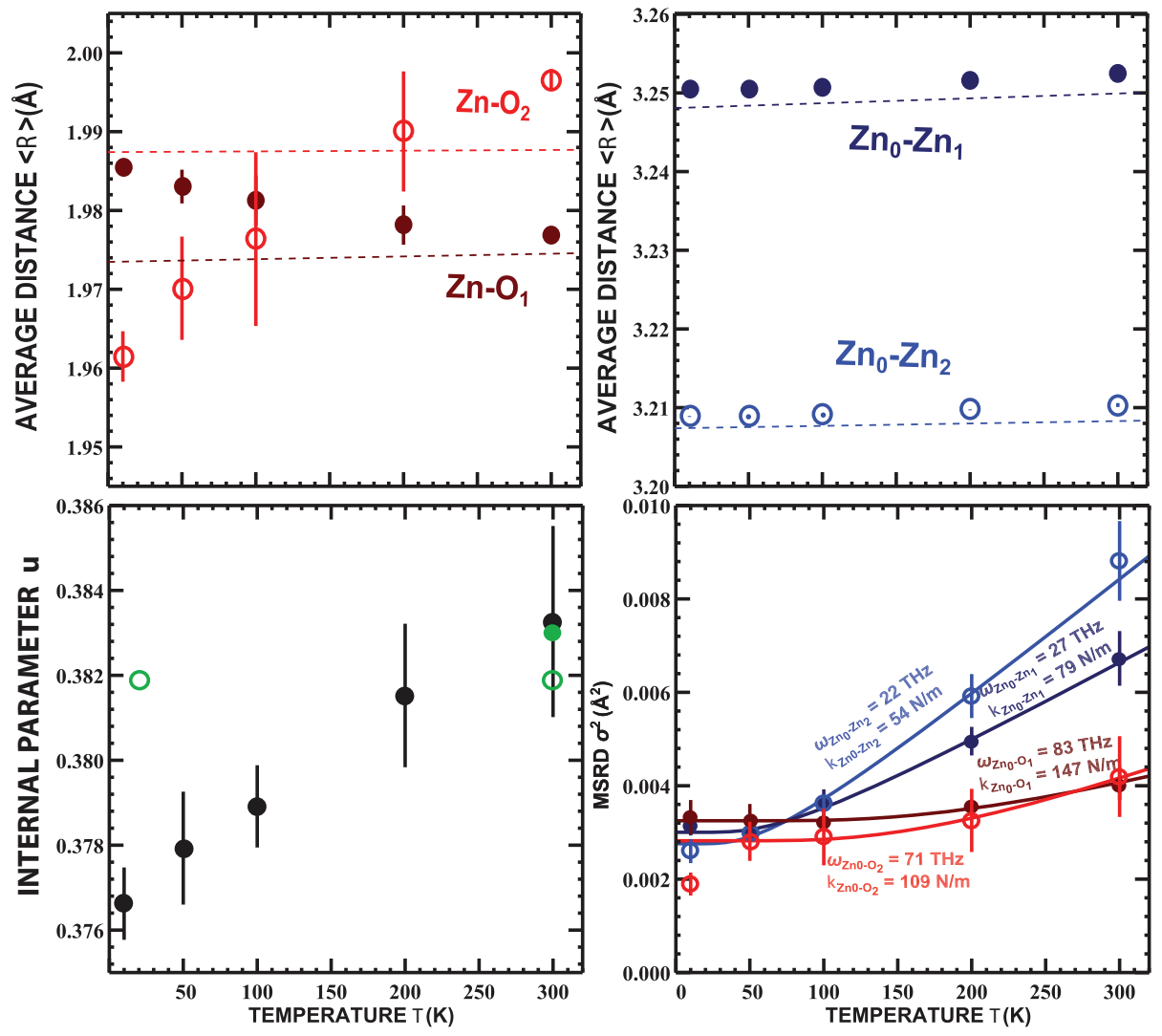

Figure 8: (Color online) Upper panels: temperature dependencies of $\mathrm{Zn}-\mathrm{O}$ (left panel) and Zn-Zn (right panel) average interatomic distances; dashed lines show x-ray diffraction data in the range from 4.2 to $300 \mathrm{~K}$ from $[10,11]$. Lower left panel: temperature dependence of the internal parameter $u$ of the oxygen Wyckoff position (2b), obtained from our RMC/EA simulations; the values of $u$ obtained from diffraction studies are shown by green empty circles (neutron diffraction [17]) and green filled circle (x-ray diffraction [16]). Lower right panel: MSRD factors as a function of temperature for the four atom pairs $\left(\mathrm{Zn}_{0}-\mathrm{O}_{1}, \mathrm{Zn}_{0}{ }^{-}\right.$ $\mathrm{O}_{2}, \mathrm{Zn}_{0}-\mathrm{Zn}_{1}$ and $\mathrm{Zn}_{0}-\mathrm{Zn}_{2}$ ) in crystalline $\mathrm{ZnO}$ (solid lines - fits by the Einstein model). 